\title{
Laparoscopic cholecystectomy after percutaneous transhepatic gallbladder drainage for acute cholecystitis in a patient with a left ventricle assist device: a case report and brief review of the literature (with video)
}

Takehiko Hanaki $^{1}$, Teppei Sunaguchi ${ }^{1}$, Keisuke Goto ${ }^{1}$, Masaki Morimoto ${ }^{1}$, Yuki Murakami $^{1}$, Naruo Tokuyasu ${ }^{1}$, Shuichi Takano ${ }^{1}$, Teruhisa Sakamoto ${ }^{1}$, Toshimichi Hasegawa $^{1}$, and Yoshiyuki Fujiwara ${ }^{1}$

${ }^{1}$ Tottori University Faculty of Medicine Graduate School of Medicine

March 7, 2022

\begin{abstract}
For acute cholecystitis in patients with left ventricular assist devices, the use of percutaneous transhepatic gallbladder drainage to calm inflammation before planned laparoscopic cholecystectomy may be helpful in safely adjusting anticoagulation and in performing safe laparoscopic cholecystectomy, as demonstrated in this case.
\end{abstract}

Title: Laparoscopic cholecystectomy after percutaneous transhepatic gallbladder drainage for acute cholecystitis in a patient with a left ventricle assist device: a case report and brief review of the literature (with video)

Authors and Affiliations : Takehiko Hanaki, Teppei Sunaguchi, Keisuke Goto, Masaki Morimoto, Yuki Murakami, Naruo Tokuyasu, Shuichi Takano, Teruhisa Sakamoto, Toshimichi Hasegawa, and Yoshiyuki Fujiwara.

Department of Gastrointestinal and Pediatric Surgery, School of Medicine, Tottori University Faculty of Medicine, Yonago, Japan

Corresponding author: Takehiko Hanaki, MD, PhD (ORCID https://orcid.org/0000-0002-4008-0207)

Department of Gastrointestinal and Pediatric Surgery, School of Medicine, Tottori University Faculty of Medicine, Yonago, Japan

Address : 36-1, Nishi-cho, Yonago, Tottori, Japan

Tel : +81-859-38-6567

Email :hanaki-ttr@umin.ac.jp

Key words: Left ventricular assist device, Laparoscopic cholecystectomy, Acute cholecystitis, Percutaneous transhepatic gallbladder drainage

\section{Abstract}

For acute cholecystitis in patients with left ventricular assist devices, the use of percutaneous transhepatic gallbladder drainage to calm inflammation before planned laparoscopic cholecystectomy may be helpful in 
safely adjusting anticoagulation and in performing safe laparoscopic cholecystectomy, as demonstrated in this case.

\section{Key Clinical Message}

For acute cholecystitis in patients with left ventricular assist devices, the use of percutaneous transhepatic gallbladder drainage to calm inflammation before planned laparoscopic cholecystectomy may be helpful, as demonstrated in this case.

\section{Introduction}

To facilitate blood circulation in the failing heart, the left ventricle assist device (LVAD) was developed and used as destination therapy. The LVAD is an alternative to heart transplantation and can be a bridge to recovery or a bridge to heart transplantation ${ }^{1-3}$ in patients with left ventricular failure. The use of LVADs prolongs survival in patients with heart failure ${ }^{3}$. In Japan, LVADs became a health insurance option for patients on the heart transplant waiting list in 2011. However, the number of patients on the transplant waiting list and, consequently, the number of patients with LVADs is increasing due to the continuing shortage of donor hearts ${ }^{3-5}$. Adequate anticoagulation is essential in patients with LVADs, as clots inside the LVAD can be fatal. However, anticoagulation can lead to intraoperative and postoperative bleeding when patients with LVADs require surgery.

Acute cholecystitis is a common abdominal condition that requires urgent intervention. Several reports describe the use of endoscopic surgery to treat cholecystitis in patients with LVADs ${ }^{6} 7$. Here, we report the use of percutaneous transhepatic gallbladder drainage (PTGBD) to relieve local inflammation due to acute cholecystitis in a LVAD transplant recipient with unstable vital status. The PTGBD was followed by an elective laparoscopic cholecystectomy (LC). An intraoperative video is provided (Additional File 1: Video S1).

\subsection{Case presentation}

A 64-year-old male on the heart transplant waiting list was admitted to the Cardiovascular Surgery ward of our hospital for intracerebral hemorrhage. He had undergone LVAD (HeartMate II, Thoratec Corporation, Pleasanton, CA, USA) implantation at 59 years of age due to dilated cardiomyopathy causing left ventricle failure. The subcortical hemorrhage was treated conservatively and the patient was rehabilitated in the same ward without any complications. However, the patient developed fever, nausea, and right upper quadrant pain with shock vitals. Computed tomography revealed an enlarged gallbladder with a thickened wall and surrounding fatty tissue opacity. He was diagnosed with acute cholecystitis and was referred to our department. Emergent surgery was considered. However, conditions were not favorable due to the infection and heart failure with an LVAD. Thus, PTGBD was performed to reduce inflammation in the gallbladder. The cholecystitis resolved in a few days. An elective LC was planned for 3 months after the PTGBD, to avoid the risk of recurrent cholecystitis that could cause LVAD infection.

The patient was taking $3.5 \mathrm{mg} / \mathrm{d}$ of warfarin with a prothrombin time-international normalized ratio of 2.0-3.0 because of the LVAD. In preparation for surgery, the anticoagulation was reversed with prothrombin complex concentrate. After induction of general anesthesia, markings were made on the skin along the subcutaneous driveline to avoid injury during trocar insertion (Figure 1). The first endoscopic trocar was inserted via the open method ( $15 \mathrm{~mm}$ vertical incision at the umbilicus). The pneumoperitoneum pressure was set at $10 \mathrm{mmHg}$ and a $30^{\circ}$ scope was inserted. The other trocars were placed as demonstrated in Figure 1. No adhesions were found around the LVAD, which was placed in the preperitoneal space. However, some adhesions were found around the gallbladder (Figure 2a, b, Additional File 1: Video S1). The neck of the gallbladder was thickened and a sub-total resection was performed. To ensure that there was no postoperative bleeding, a drain was placed in the liver bed and the operation was completed. The duration of surgery was $114 \mathrm{~min}$, and the estimated amount of blood loss was $5 \mathrm{~mL}$.

\subsection{Postoperative clinical course}


The patient was returned to the intensive care unit and anticoagulants were restarted on postoperative day (POD) 1 with 10,000 units/d of heparin and $3 \mathrm{mg} / \mathrm{d}$ of warfarin. On POD3, prolonged coagulation was observed and heparin was terminated (Figure 3). The patient was transferred from the intensive care unit to the Cardiovascular Surgery ward on the same day. The postoperative course was uneventful. No LVAD related problems occurred and no postoperative blood transfusion was required. 10 months after the LC, the patient is on the waiting list for a heart transplant.

\section{Discussion}

According to our experience and previous reports, the laparoscopic approach to cholecystectomy may be useful ${ }^{6}$. Pneumoperitoneum may increase the preload on the heart and cause vital changes in LVAD patients ${ }^{8}$. However, in this case, no intraoperative change due to pneumoperitoneum was observed. In the present study, we performed pneumoperitoneum at $10 \mathrm{mmHg}$. However, previous reports suggest that a pneumoperitoneum at $10-15 \mathrm{mmHg}$ is also acceptable (Table 1) ${ }^{69-23}$.

There are currently nine case reports and seven case series in the literature, including 32 cases that described LC (Table 1). Of the 42 total cases (including the presented case), only one case $(2.4 \%)$ converted to open surgery. Nearly half of the patients $(40.5 \%)$ underwent scheduled elective surgery. This trend seems to be particularly strong since 2015. We speculate that this change is partly related to increased awareness of the acute cholecystitis guidelines ${ }^{24}$. i.e., the use of alternative methods such as gallbladder drainage rather than emergency surgery in the case of organ failure. However, the rate of PTGBD was not stated in many of the previous reports; therefore, the overall rate is unknown. The safety and efficacy of PTGBD have been confirmed by many case-control studies in patients with cholecystitis. PTGBD is recommended as a standard drainage method for patients with acute cholecystitis at high surgical risk ${ }^{24}$. In this case, the following factors contributed to our success: 1 ) the acute cholecystitis was treated with PTGBD to reduce inflammation before the scheduled surgery, 2) time was given before the scheduled surgery to consider warfarin antagonism, and 3) the surgery was performed in a calm circulatory state. In our case, the operation was completed safely, with lower blood loss compared to the blood loss in previous reports. Although our institution is not a heart transplant hospital, LVAD transplantation is performed in the Department of Cardiovascular Surgery at Tottori University Hospital. Patients with LVADs are admitted to our hospital. As the number of patients with LVADs is increasing worldwide, more cases of acute abdominal problems in patients after LVAD transplantation will be encountered. The use of PTGBD to reduce the cholecystitis before performing the LC is beneficial.

In conclusion, we described the case of a patient with end-stage heart failure and an implanted LVAD who was diagnosed with acute cholecystitis. The patient was successfully and safely treated with PTGBD followed by elective LC. In patients with LVADs who develop acute cholecystitis, the use of early PTGBD to eliminate inflammation in the biliary tract is useful. Awaiting surgery prevents fatal complications, such as perioperative bleeding and biliary tract injury.

\section{List of abbreviations}

LVAD, Left ventricular assist devices; PTGBD, Percutaneous transhepatic gallbladder drainage; LC, Laparoscopic cholecystectomy

\section{Availability of data and materials}

The relevant data and images related to the patient's course and care are included in the article.

\section{Conflict of interests}

Authors have no conflicts of interest or financial ties to disclose.

\section{Funding}

This report did not receive any specific grant from funding agencies in the public, commercial, or not-forprofit sectors. 


\section{Consent for publication}

Written informed consent was obtained from the patient for the publication of this case report and accompanying images. A copy of written consent is available for review by the Editor-in-Chief of this journal.

\section{Authors' contributions}

TaH: collected the patient data, performed surgery and a literature review, and wrote the manuscript. TS, KG, MM, YM, NT, ST, and TS: revised the manuscript. ToH and YF: were involved in overall supervision of the study. All authors have read and approved the final version of the manuscript.

\section{References}

1. Angermayr L, Velasco Garrido M, Busse R. 2007. Ventricular assist devices for heart failure. GMS Health Technol Assess 3: Doc10.

2. Iglesias-Álvarez D, Pathania V. 2021. LVAD as a bridge to decision complicated with pump thrombosis and infection. Indian J Thorac Cardiovasc Surg 37: 341-344.

3. Ben Gal T, Ben Avraham B, Milicic D, et al. 2021. Guidance on the management of left ventricular assist device (LVAD) supported patients for the non-LVAD specialist healthcare provider: executive summary. European Journal of Heart Failure 23: 1597-1609.

4. Sakaguchi T, Matsumiya G, Yoshioka D, et al. 2013. DuraHeart magnetically levitated left ventricular assist device: Osaka University experience. Circ J 77: 1736-1741.

5. Li X, Kondray V, Tavri S, et al. 2019. Role of imaging in diagnosis and management of left ventricular assist device complications. Int J Cardiovasc Imaging 35: 1365-1377.

6. Naitoh T, Morikawa T, Sakata N, Unno M, Akiyama M, Saiki Y. 2013. Emergency laparoscopic cholecystectomy for a patient with an implantable left ventricular assist device: report of a case. Surg Today 43 : 313-316.

7. Chinen Y, Ogino T, Fujino S, et al. 2021. A case of single-incision laparoscopic surgery for acute appendicitis with left ventricular assist device. Asian J Endosc Surg 14: 607-610.

8. Hurlburt L, Roscoe A, van Rensburg A. 2014. The use of prothrombin complex concentrates in two patients with non-pulsatile left ventricular assist devices. J Cardiothorac Vasc Anesth 28: 345-346.

9. Votapka TV, Pennington DG, McBride LR, Kaminski DL, Andrus CH, Swartz MT. 1994. Noncardiac operations in patients supported with mechanical circulatory support devices. J Am Coll Surg 179: 318-320.

10. Eckhauser AE, Melvin WV, Sharp KW. 2006. Management of general surgical problems in patients with left ventricular assist devices. Am Surg 72: 158-161.

11. Nissen NN, Korman J, Kleisli T, Magliato KE. 2005. Laparoscopic cholecystectomy in a patient with a biventricular cardiac assist device. Jsls 9: 481-484.

12. Kartha V, Gomez W, Wu B, Tremper K. 2008. Laparoscopic cholecystectomy in a patient with an implantable left ventricular assist device. Br J Anaesth 100: 652-655.

13. Livi U, Guzzi G, Tursi V, et al. 2009. Laparoscopic approach for urgent abdominal surgery in patients with left ventricular assist devices. J Cardiovasc Med (Hagerstown) 10: 741-744.

14. Amir O, Bitterman A, Eden A. 2012. Laparoscopic cholecystectomy in a left ventricular assist devicesupported patient. Isr Med Assoc J 14: 525-526.

15. Eck DL, Belli EV, Smith CD, Stauffer JA. 2014. Laparoscopic cholecystectomy in patients with HeartMate II left ventricular assist devices. J Laparoendosc Adv Surg Tech A 24: 100-103. 
16. Reich H, Ramzy D, Czer L, et al. 2015. Hemodynamic Consequences of Laparoscopy for Patients on Mechanical Circulatory Support. J Laparoendosc Adv Surg Tech A 25: 999-1004.

17. Yoon AJ, Sohn J, Grazette L, Fong MW, Bowdish ME. 2016. Pan-Cardiac Cycle Fixed Mitral Valve Opening in an LVAD Patient Presenting with Hemorrhagic Shock. Echocardiography 33: 644-646.

18. Ashfaq A, Chapital AB, Johnson DJ, Staley LL, Arabia FA, Harold KL. 2016. Safety and Feasibility of Laparoscopic Abdominal Surgery in Patients With Mechanical Circulatory Assist Devices. Surg Innov 23: 469-473.

19. Suresh V, Bishawi M, Bryner B, et al. 2019. Outcomes of Laparoscopic Cholecystectomy in Patients Supported with a Left Ventricular Assist Device. J Laparoendosc Adv Surg Tech A 29: 441-444.

20. Vigneswaran Y, Wang V, Krezalek M, et al. 2019. Laparoscopic procedures in patients with cardiac ventricular assist devices. Surg Endosc 33: 2181-2186.

21. Takagi A, Nagai E, Toda T, et al. 2019. Emergency laparoscopic cholecystectomy for intraabdominal hemorrhage in a patient with a left ventricular assist device: a case report. Surg Case Rep 5: 196.

22. Zilbermints V, Israeli O, Ben Abraham B, et al. 2020. Abdominal Surgery in Patients with a Ventricular Assist Device: A Single Center Experience in Israel. Isr Med Assoc J 22: 369-373.

23. Beetz O, Bajunaid A, Meissler L, et al. 2020. Abdominal Surgery in Patients with Ventricular Assist Devices: a Single-Center Report. Asaio j 66: 890-898.

24. Mayumi T, Okamoto K, Takada T, et al. 2018. Tokyo Guidelines 2018: management bundles for acute cholangitis and cholecystitis. J Hepatobiliary Pancreat Sci 25: 96-100.

Figure legends

Figure 1. Postoperative abdominal photograph showing the port placement and the position of the subcutaneous driveline markings.

Figure 2. Intraoperative pictures showing the left ventricle assist device.

(a) The left subcostal preperitoneal space, (b) adhesion around the gallbladder, and (c) the liver bed after cholecystectomy.

Figure 3. Perioperative anticoagulation management.

PCC, prothrombin complex concentrate; POD, postoperative day

Table 1. Reports of laparoscopic cholecystectomy in patients with left ventricular assist devices.

Hosted file

image1.emf available at https://authorea.com/users/463891/articles/558909-laparoscopiccholecystectomy-after-percutaneous-transhepatic-gallbladder-drainage-for-acute-

cholecystitis-in-a-patient-with-a-left-ventricle-assist-device-a-case-report-and-briefreview-of-the-literature-with-video

Additional File 1: Video S1. Intraoperative video 
Fig. 1

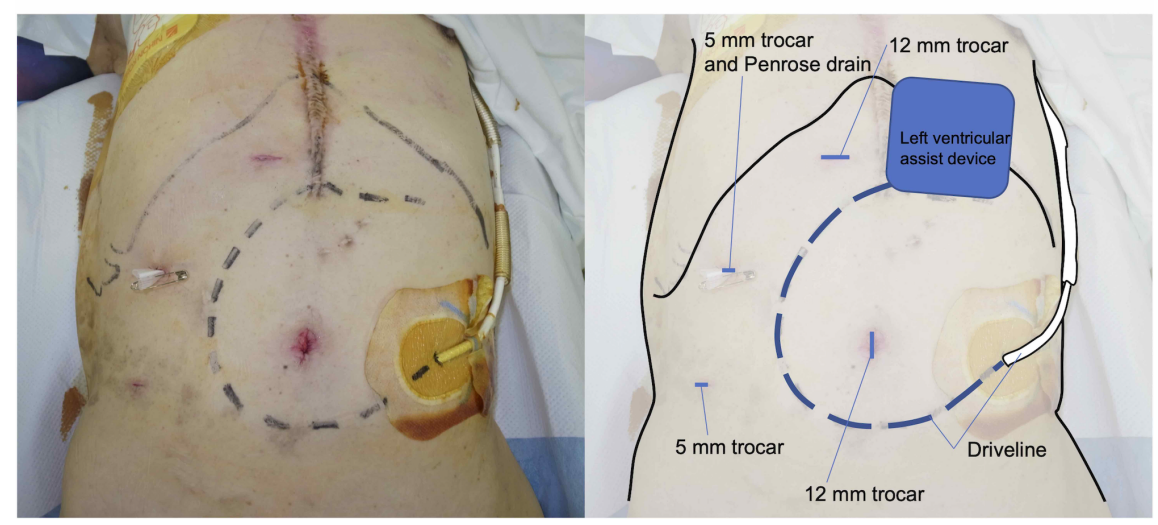

Fig. 2

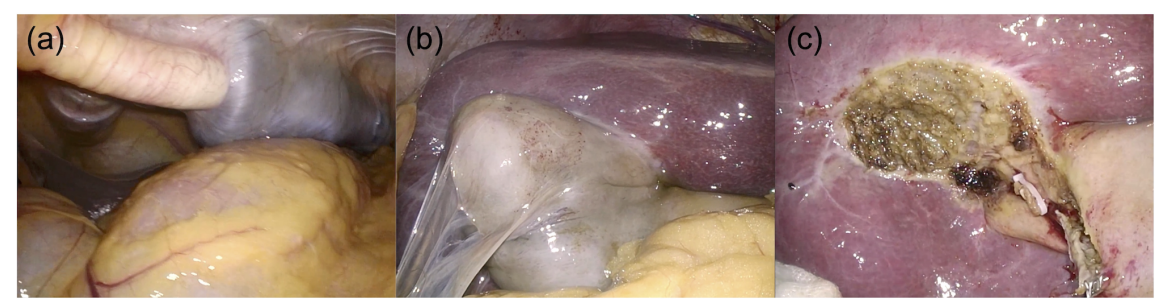

Fig. 3

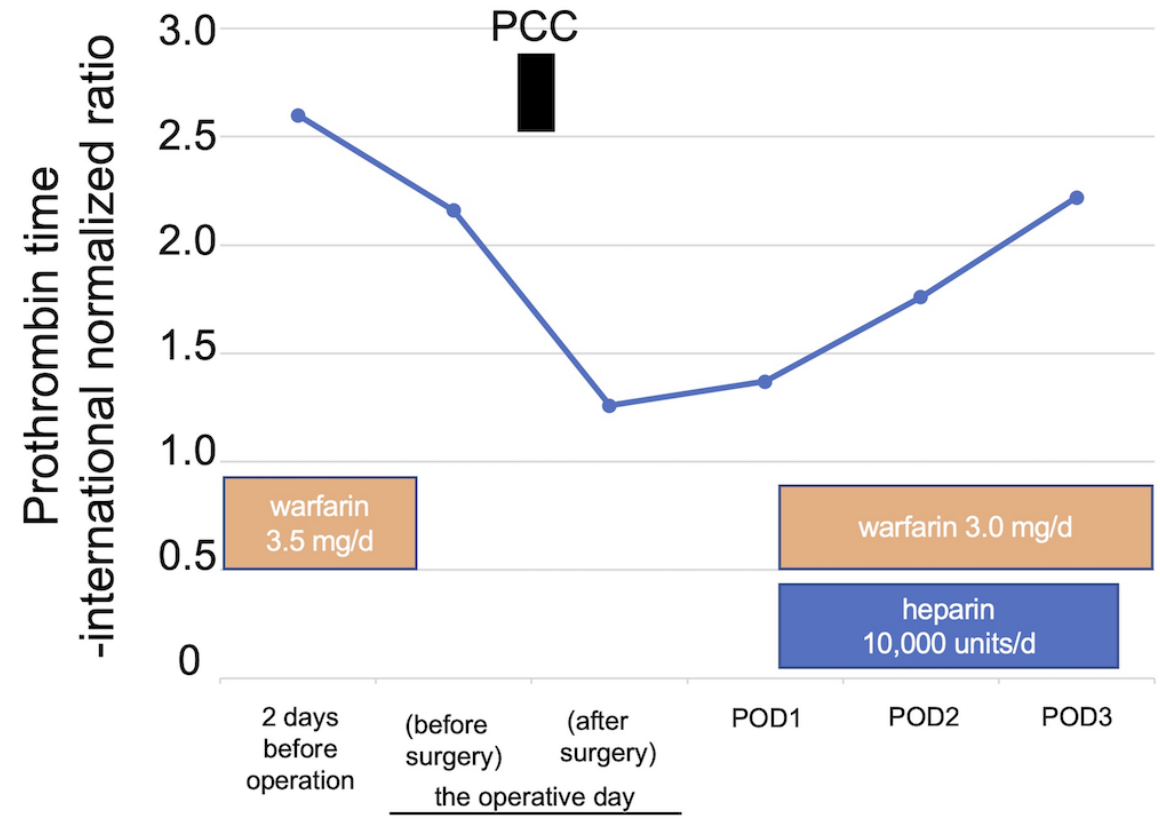

